\title{
8th edition AJCC/UICC staging of cancers of the esophagus and esophagogastric junction: application to clinical practice
}

\author{
Thomas W. Rice ${ }^{1}$, Deepa T. Patil ${ }^{2}$, Eugene H. Blackstone ${ }^{1,3}$ \\ ${ }^{1}$ Department of Thoracic and Cardiovascular Surgery, Heart and Vascular Institute, ${ }^{2}$ Department of Pathology, Pathology and Laboratory Medicine \\ Institute, ${ }^{3}$ Department of Quantitative Health Sciences, Research Institute, Cleveland Clinic, Cleveland, Ohio, USA \\ Correspondence to: Thomas W. Rice, MD. Department of Thoracic and Cardiovascular Surgery, Cleveland Clinic, 9500 Euclid Avenue/Desk JJ-40, \\ Cleveland, OH 44195, USA. Email: ricet@ccf.org.
}

\begin{abstract}
The 8th edition of the American Joint Committee on Cancer (AJCC) staging of epithelial cancers of the esophagus and esophagogastric junction (EGJ) presents separate classifications for clinical (cTNM), pathologic (pTNM), and postneoadjuvant (ypTNM) stage groups. Histopathologic cell type markedly affects survival of clinically and pathologically staged patients, requiring separate groupings for each cell type, but ypTNM groupings are identical for both cell types. Clinical categories, typically obtained by imaging with minimal histologic information, are limited by resolution of each method. Strengths and shortcomings of clinical staging methods should be recognized. Complementary cytology or histopathology findings may augment imaging and aid initial treatment decision-making. However, prognostication using clinical stage groups remains coarse and inaccurate compared with pTNM. Pathologic staging is losing its relevance for advanced-stage cancer as neoadjuvant therapy replaces esophagectomy alone. However, it remains relevant for early-stage cancers and as a staging and survival reference point. Although pathologic stage could facilitate decision-making, its use to direct postoperative adjuvant therapy awaits more effective treatment. Prognostication using pathologic stage groups is the most refined of all classifications. Postneoadjuvant staging (ypTNM) is introduced by the AJCC but not adopted by the Union for International Cancer Control (UICC). Drivers of this addition include absence of equivalent pathologic (pTNM) categories for categories peculiar to the postneoadjuvant state (ypT0N0-3M0 and ypTisN0-3M0), dissimilar stage group compositions, and markedly different survival profiles. Thus, prognostication is specific for patients undergoing neoadjuvant therapy. The role of ypTNM classification in additional treatment decision-making is currently limited. Precision cancer care advances are necessary for this information to be clinically useful.
\end{abstract}

Keywords: Clinical stage; pathologic stage; postneoadjuvant stage; decision-making; prognostication; precision cancer care

Submitted Dec 08, 2016. Accepted for publication Mar 07, 2017.

doi: 10.21037/acs.2017.03.14

View this article at: http://dx.doi.org/10.21037/acs.2017.03.14

\section{Introduction}

Staging of cancer of the esophagus and esophagogastric junction (EGJ), presented in chapter 16 of the 8 th edition American Joint Committee on Cancer (AJCC) Cancer Staging Manual (1), was derived from a machine-learning analysis of data from six continents from the Worldwide Esophageal Cancer Collaboration (WECC) (2-7). The purpose of this manuscript is to review staging in the 8th edition, which now includes clinical (cTNM), pathologic
(pTNM), and postneoadjuvant therapy (ypTNM) stage groupings, and examine its application to clinical practice.

\section{8th edition clinical staging}

TNM categories-the facts about cancer-are judged clinically (cTNM) based on imaging studies, with minimal histologic information. This distinguishes them from pathologic cancer facts (pTNM) obtained by and large 
through microscopic examination of resection specimens. These TNM cancer facts (Table 1) are central to treatment decision-making and rely on cTNM being an accurate reflection of pTNM.

Stage groups are coarse prognostic collections based on an amalgamation of cancer facts. That prognostication based on cTNM differs from that based on pTNM reflects inaccuracies of obtaining cancer facts by current clinical staging modalities. These inaccuracies result in dissimilar stage group composition and survival profiles of cTNM $v s$. pTNM groups $(2,5-7)$.

Histopathologic cell type markedly affects survival of cTNM-staged patients. Survival of early- and intermediatestage patients is worse for those with squamous cell carcinoma than those with adenocarcinoma, necessitating separate stage grouping by cell type.

Histologic grade (G) markedly affects survival of patients with cT1-2N0M0 adenocarcinomas and cT2N0M0 squamous cell carcinomas. The AJCC Upper Gastrointestinal Expert Panel, concerned about the accuracy of G on biopsy, eliminated $\mathrm{G}$ from 8th edition cTNM stage groups, with the expectation that it would be reexamined for the 9th edition. Thus, although cTNM stage groups have been introduced into the 8th edition, they are more for coarse prognostication rather than for decision-making (Table 2).

\section{Applications of 8th edition clinical staging (cTNM) to clinical practice}

Clinical staging is limited by resolution of imaging methods. Strengths and limitations of each method should be taken into account when interpreting clinical staging.

\section{Location (cL)}

Assessment of cancer location (cL) made during esophagoscopy is crucial (1). The definition of $\mathrm{cL}$ has changed from the position of the upper edge of the cancer (7th edition) to its epicenter (8th edition), both referenced to distance from the incisors. Clinically, the epicenter is determined from upper and lower border measurements, which also provide cancer length. For treatment planning, it is critical to know the upper border for cancers of the cervical and upper thoracic esophagus and the lower border for cancers of the lower thoracic esophagus and EGJ. Alternatively, $\mathrm{cL}$ may be determined from chest computed tomography (CT) (1).

EGJ staging has been limited by reliance on simple measurements to determine whether an adenocarcinoma is esophageal or gastric. Conflicting statistical analyses necessitated a "place card" consensus decision; thus, the EGJ was redefined in the 8th edition: adenocarcinomas with epicenters no more than $2 \mathrm{~cm}$ into the gastric cardia are staged as esophageal adenocarcinomas, and those extending further are staged as stomach cancers (1). The genetic signature of EGJ cancers may be more accurate in identifying the cell of origin for cancer staging rather than its gross location $(8,9)$. Cancer genetics will be a subsequent focus of the 9th edition staging of EGJ cancers.

\section{Histologic cell type}

Biopsy is mandatory and is the principal means of determining cell type. Because obliterative neoadjuvant therapy or endoscopic resection may prohibit future assessment of the primary cancer, this biopsy may provide the only facts about the cancer.

In most instances, classifying cancers as squamous cell carcinoma or adenocarcinoma relies on identifying features of squamous differentiation (keratin pearl formation, intercellular bridges, and cells with abundant glassy eosinophilic cytoplasm) versus gland formation for adenocarcinoma. However, this distinction can be challenging in specimens with limited diagnostic material and in higher $\mathrm{G}$ cancers. Ancillary markers, such as p63, p40, and cytokeratin $5 / 6$ for squamous differentiation, and Alcian blue-PAS stain to demonstrate subtle intracellular mucin for adenocarcinoma, can be helpful.

$c G$

cG is important for treatment decisions regarding cT1$2 \mathrm{~N} 0 \mathrm{M} 0$ adenocarcinomas and cT2N0M0 squamous cell carcinomas and is a predictor of survival. Unfortunately, it is inconsistently reported in biopsy specimens, because superficial biopsy samples may provide limited material to accurately grade cancer differentiation.

Additionally, reporting $\mathrm{cG}$ has not been required previously for biopsy specimens (Table 1). Every attempt should be made to grade cancers using criteria outlined by the World Health Organization $(10,11)$. Low-grade (G1) and moderately differentiated cancers (G2) are likely subject to substantial interobserver variability. However, poor differentiation or signet-ring cell morphology (G3) are associated with poor outcome (12) and thus must be documented in the biopsy pathology report as cG.

$c T$

Esophageal ultrasound (EUS) provides detailed examination 


\begin{tabular}{ll}
\multicolumn{2}{l}{ Table 1 Cancer staging categories for cancer of the esophagus and esophagogastric junction } \\
\hline Category & Criteria \\
\hline T category & Tumor cannot be assessed \\
T0 & No evidence of primary tumor \\
Tis & High-grade dysplasia, defined as malignant cells confined by the basement membrane \\
T1 & Tumor invades the lamina propria, muscularis mucosae, or submucosa \\
T1 & Tumor invades the lamina propria or muscularis mucosae \\
T1 & Tumor invades the submucosa \\
T2 & Tumor invades the muscularis propria \\
T3 & Tumor invades adventitia \\
T4 & Tumor invades adjacent structures \\
T4a* & Tumor invades the pleura, pericardium, azygos vein, diaphragm, or peritoneum \\
T4b* & Tumor invades other adjacent structures, such as aorta, vertebral body, or trachea
\end{tabular}

\section{$\mathrm{N}$ category}

NX Regional lymph nodes cannot be assessed

No No regional lymph node metastasis

N1 Metastasis in 1-2 regional lymph nodes

N2 Metastasis in 3-6 regional lymph nodes

N3 Metastasis in 7 or more regional lymph nodes

$\begin{array}{ll}\text { M category } & \\ \text { M0 } & \text { No distant metastasis } \\ \text { M1 } & \text { Distant metastasis }\end{array}$

Adenocarcinoma G Category

GX Differentiation cannot be assessed

G1 Well differentiated. $>95 \%$ of tumor is composed of well-formed glands

G2 Moderately differentiated. $50 \%$ to $95 \%$ of tumor shows gland formation

G3 $^{\dagger} \quad$ Poorly differentiated. Tumors composed of nest and sheets of cells with $<50 \%$ of tumor demonstrating glandular formation
Squamous cell carcinoma G category
GX Differentiation cannot be assessed cells. Tumor cells are arranged in sheets, and mitotic counts are low
G2 Moderately differentiated. Variable histologic features, ranging from parakeratotic to poorly keratinizing lesions. Generally, pearl formation is absent
G3 $^{\ddagger} \quad$ Poorly differentiated. Consists predominantly of basal-like cells forming large and small nests with frequent central by small numbers of parakeratotic or keratinizing cells
Squamous cell carcinoma $L$ category***
LX Location unknown
Upper Cervical esophagus to lower border of azygos vein
Middle Lower border of azygos vein to lower border of inferior pulmonary vein
Lower Lower border of inferior pulmonary vein to stomach, including esophagogastric junction

G1 Well-differentiated. Prominent keratinization with pearl formation and a minor component of nonkeratinizing basal-like necrosis. The nests consist of sheets or pavement-like arrangements of tumor cells, and occasionally are punctuated

${ }^{*}$, subcategories; ${ }^{\dagger}$, if further testing of "undifferentiated" cancers reveals a glandular component, categorize as adenocarcinoma G3; ${ }^{\ddagger}$, if further testing of "undifferentiated" cancers reveals a squamous cell component, or if after further testing they remain undifferentiated, categorize as squamous cell carcinoma G3; ${ }^{* \star *}$, location is defined by epicenter of esophageal tumor. 


\begin{tabular}{|c|c|c|c|}
\hline cStage group & cT & $\mathrm{cN}$ & $\mathrm{cM}$ \\
\hline \multicolumn{4}{|c|}{ Squamous cell carcinoma } \\
\hline 0 & Tis & NO & Mo \\
\hline I & $\mathrm{T} 1$ & No-1 & MO \\
\hline \multirow[t]{2}{*}{ II } & T2 & No-1 & MO \\
\hline & T3 & NO & MO \\
\hline \multirow[t]{2}{*}{ III } & T3 & $\mathrm{N} 1$ & MO \\
\hline & $\mathrm{T} 1-3$ & N2 & MO \\
\hline \multirow[t]{2}{*}{ IVA } & $\mathrm{T} 4$ & No-2 & MO \\
\hline & $\mathrm{T} 1-4$ & N3 & MO \\
\hline IVB & $\mathrm{T} 1-4$ & No-3 & M1 \\
\hline \multicolumn{4}{|c|}{ Adenocarcinoma } \\
\hline 0 & Tis & NO & MO \\
\hline I & $\mathrm{T} 1$ & NO & MO \\
\hline IIA & $\mathrm{T} 1$ & N1 & MO \\
\hline IIB & T2 & No & MO \\
\hline \multirow[t]{2}{*}{ III } & $\mathrm{T} 2$ & $\mathrm{~N} 1$ & MO \\
\hline & T3-4a & No-1 & M0 \\
\hline \multirow[t]{3}{*}{ IVA } & $\mathrm{T} 1-4 \mathrm{a}$ & N2 & MO \\
\hline & $\mathrm{T} 4 \mathrm{~b}$ & No-2 & Mo \\
\hline & $\mathrm{T} 1-4$ & N3 & MO \\
\hline IVB & $\mathrm{T} 1-4$ & No-3 & M1 \\
\hline
\end{tabular}

of the esophageal wall and currently is the procedure of choice for determining $\mathrm{c} T$. The esophageal wall is viewed as alternating hyperechoic (white) and hypoechoic (black) layers on EUS. The muscularis propria, imaged as the fourth layer (hypoechoic), is vital in differentiating T1, T2, and $\mathrm{T} 3$ cancers. Hypoechoic cancers are c $\mathrm{T} 1$ if there is no invasion of the fourth layer, cT2 if invasion is into the fourth layer, or cT3 if invasion is beyond the fourth layer. Additionally, EUS is used to evaluate the interface (between 4 th and 5 th layers) between the primary cancer and adjacent structures. If invasion of the fifth layer is detected, the cancer is cT4.

The performance index for distinguishing $\mathrm{T} 1$ or $\mathrm{T} 2$ cancers from $\mathrm{T} 3$ or T4 cancers by EUS is 0.89 for esophageal cancers and 0.91 for EGJ cancers (13). This distinction is essential for decision-making: typically,
T3-4 cancers have a high probability of $\mathrm{N}+$ and require neoadjuvant therapy, while T1-2 cancers are likely N0, requiring resection alone (14).

The clinical categorization of cT1-2 cancer directs future therapy. At higher EUS frequencies that focus on more superficial layers, subclassification of cT1a from cT1b cancers should be possible. However, most studies have shown poor accuracy in this subclassification (15-18). EUS has been reported as unreliable in staging T2N0M0 cancers $(19,20)$; therefore, for cT1N0M0 and cT2N0M0 cancers, additional information is needed for decision-making. For T1N0M0 cancers, endoscopic mucosal resection (EMR) and endoscopic submucosal dissection (ESD) are highly effective in confirming EUS cT1N0M0 and differentiating cT1a from cT1b (18,21-23). Note that pathologically confirmed T1 by EMR remains cT1, not pT1 (24).

For cT2N0M0 cancers, EMR and enhanced imaging are currently unreliable or unavailable. The use of $\mathrm{G}$, particularly the finding of $\mathrm{cG} 3$, which is associated with reduced survival, may facilitate decision-making and prognostication $(6,21)$.

\section{$c N$}

EUS, CT, and fluorodeoxyglucose positron emission tomography (FDG-PET) afford regional lymph node imaging and are the principal non-invasive modalities for $\mathrm{cN}$ determination. Each examination indirectly assesses the potential of a lymph node harboring metastases and thus has limitations specific to each technique.

EUS is used to evaluate size, shape, border, and internal echocardiographic characteristics of regional lymph nodes. Larger, more rounded, well demarcated hypoechoic lymph nodes are most likely to contain metastasis. However, reliance on EUS imaging assessment of $\mathrm{cN}$ is problematic. In patients with a $60 \%$ prevalence of $\mathrm{pN}+$, using EUS criteria of $>5 \mathrm{~mm}$, round borders, smooth shape, and hypoechoic center for $\mathrm{cN}+$, EUS was only $20 \%$ specific for $\mathrm{N}+$, resulting in overstaging in $80 \%$ of $\mathrm{pN} 0$ cancers (25).

An enlarged lymph node on CT suggests nodal metastasis. The short axis of nodes is easily measured; intrathoracic and abdominal lymph nodes $>1 \mathrm{~cm}$ are considered enlarged (26). However, probability is small that $\mathrm{cN}$ can be determined by lymph node size alone (27). Sources of false-negative examinations are normal-sized nodes that contain metastatic deposits, and metastatic nodes in direct contact with the cancer that may be indistinguishable from it. Similarly, false-positive examinations result from non-malignant nodal enlargement, 
as seen in inflammation. In a recent review, CT was $50 \%$ sensitive (range, $41-60 \%$ ) and $83 \%$ specific (range, $77-$ $89 \%$ ) in $\mathrm{cN}$ assessment (28).

Metabolic evaluation of esophageal cancer by FDGPET relies not only on metastatic deposit size but also on intensity of FDG uptake and decay. Theoretically, it is possible to identify microscopic metastases if glucose metabolism is sufficient to concentrate large quantities of FDG. FDG-PET cannot differentiate adjacent $\mathrm{N}+$ from the primary cancer (29) and is least sensitive in assessing lymph nodes in the mid- and lower-thoracic esophagus (30). In a meta-analysis of 10 studies, FDG-PET N categorization was $57 \%$ sensitive (range, $43-70 \%$ ) and $85 \%$ specific (range, $76-95 \%$ ) (28). Because of its relatively good specificity, the main role of PET is confirmation of $\mathrm{cN} 0$ (31). Adding CT to FDG-PET increases accuracy, sensitivity, specificity, and negative predictive value (NPV) minimally (32); however, its biggest impact is in positive predictive value (PPV), with an increase from $69 \%$ to $82 \%$.

These imaging modalities have remarkably similar performance in $\mathrm{cN}$ assessment: accuracy is reported to be $66 \%$ for EUS, $63 \%$ for CT, and $68 \%$ for PET, sensitivities $42 \%, 35 \%$, and $35 \%$, respectively, and specificities $91 \%$, $93 \%$, and $87 \%$, respectively (33).

Therefore, histologic confirmation of $\mathrm{cN}$ is critical for accurate clinical staging. Endosonographic-directed fine needle aspiration (EUS-FNA) is strongly recommended by the AJCC (1). In a multicenter study of 171 patients, EUS-FNA of 192 lymph nodes was performed (34). Sensitivity, specificity, PPV, and NPV for determining $\mathrm{cN}$ was $92 \%, 93 \%, 100 \%$, and $86 \%$, respectively. In a more recent meta-analysis, EUS-FNA was $92 \%$ sensitive and $93 \%$ specific for $\mathrm{cN}$, with a PPV of $100 \%$ and an NPV of $86 \%$ (28). Specificity may be lower in FNA samples obtained from lymph nodes located adjacent to the cancer, because passage of the needle through the cancer may cause contamination (35). Inadequate specimens are obtained in $11 \%$ to $16 \%$ of EUS-FNA specimens, but cytologic examination at the time of harvesting can improve quality (36). More invasive lymph node sampling, such as by thoracoscopy, laparoscopy, or mediastinoscopy and biopsy, may be necessary to ensure optimal clinical staging. Categorization of cytologically or pathologically confirmed regional lymph node metastasis remains $\mathrm{cN}$, not $\mathrm{pN}$ (24).

Subclassification of $\mathrm{cN}+$ requires determining the number of cancer-positive regional lymph nodes. It is possible to determine this number accurately, and this clinical assessment is predictive of survival (37-39).

\section{$c M$}

The value of EUS in screening for distant metastases (cM1) is extremely limited. The distant organ must be in direct contact with the upper gastrointestinal tract for it to be useful. EUS finding of ascites is suspicious for intraperitoneal metastases.

Chest and abdomen CT with intravenous and oral contrast can be used to detect distant metastasis (cM); however, it has been reported to be only $37-66 \%$ sensitive (40). FDG-PET imaging is more accurate. In a comparison report on detecting cM1, sensitivity of FDG-PET was $69 \%$, specificity $93 \%$, and overall accuracy $84 \%$ compared with $46 \%, 74 \%$, and $63 \%$, respectively, for CT (41). FDGPET/CT imaging further increases detection of distant metastases. However, this must be balanced with more false-positive findings, resulting in unnecessary additional investigations. The clinical staging benefit of FDG-PET/ CT may be limited if comprehensive conventional staging is performed, which includes chest, abdomen, and pelvis CT, EUS, and ultrasonography of the neck (1).

Cytologic or histopathologic confirmation of cM1 is recommended by the AJCC (1). If there is pathologic confirmation of distant metastatic cancer, categorization is $\mathrm{pM} 1$, not $\mathrm{cM} 1$, in contrast to $\mathrm{cT}$ and $\mathrm{cN}(1,24)$.

\section{Clinical staging strategies}

In 2017, esophagogastroduodenoscopy (EGD) with biopsy, EMR, ESD, EUS, EUS-FNA, and FDG-PET/ CT are mainstays in clinical staging of cancer of the esophagus and EGJ. No single test is sufficient, and thus these investigations are complementary. The order in which they are used is variable and depends on availability, cost, scheduling, and patient and physician preference. There are two common strategies. The first is EGD with biopsy/EMR/ESD, EUS, and EUS-FNA to determine cell type, cG, cT, and cN, followed by FDG-PET/CT for additional $\mathrm{cN}$ and $\mathrm{cM}$ staging. The endoscopic procedures can be performed at one sitting, ensuring that data for all staging categories are complete. This strategy is efficient, but costly. The second strategy begins with CT or FDGPET/CT evaluation for $\mathrm{cM} 1$ and no further testing if M1 is confirmed. For cM0 cancers, clinical staging addresses the other categories necessary to prescribe therapy. This sequential strategy is cost effective but inefficient and does not ensure that all clinical staging categories are determined (missing cTNM data). The modalities used for clinical staging must therefore be recorded in the patient's medical record (Box 1). 


\begin{tabular}{l} 
Box 1 Registry data collection variables \\
\hline Clinical staging modalities (EGJ and biopsy, EUS, EUS-FNA, CT, \\
PET/CT) \\
\hline Tumor length \\
\hline Depth of invasion \\
\hline Number of nodes involved, clinical \\
\hline Number of nodes involved, pathological \\
\hline Location of nodal disease, clinical \\
\hline Location of nodal disease, pathological \\
\hline Sites of metastasis, if applicable \\
\hline Presence of skip lesions: T(m) \\
\hline Perineural invasion \\
\hline LVI (lymphatic, vascular, both) \\
\hline Extranodal extension \\
\hline Type of surgery \\
\hline Chemotherapy \\
\hline Chemoradiation therapy (for ypTNM) \\
\hline Surgical margin (negative R0, microscopic+R1, \\
macroscopic+R2) \\
\hline HER2 status (positive or negative) for adenocarcinoma \\
\hline EGJ, esophagogastric junction; EUS, esophageal ultrasound; \\
LVI, lymphovascular invasion. \\
\hline
\end{tabular}

\section{Clinical staging: decision-making}

Clinical staging facilitates decision-making and has the potential to provide precision cancer care. However, as described above, clinical stage may not reflect pathologic stage. Therefore, every effort must be made to add complementary information to increase accuracy of clinical staging categories. Clinically staged cT1N0M0 and cT2N0M0 cancers can be further studied and staging information augmented by adding $\mathrm{cG}$ and EMR results. Patients with $\mathrm{cN} 1$ and $\mathrm{cM} 1$ cancers will benefit from histologic confirmation of advanced cancer. The future of decision-making relies on new clinical staging modalities and cancer-specific therapy.

\section{Clinical staging: prognostication}

Survival according to cTNM stage groups is "pinched together" compared with pathologic stage groups $(2,3,5,6)$.
This results from multiple factors, including failure to use, or ineffectual use of, clinical staging modalities, understaging of "early" clinical cancers, overstaging of "advanced" clinical cancers, and unpredictability of effectiveness of neoadjuvant treatment (downstaging) of advanced cancers. This results in "regression toward the mean" of survival for cancers, with vastly different survival based on pTNM. Thus, prognostication using clinical stage groups is coarse and may be inaccurate.

\section{8th edition pathologic staging}

Cancer staging was initially proposed for "cases not previously treated and that the extent of the disease must be determined and recorded on clinical examination only" (42). However, inaccuracies of clinical staging and pathologic assessment after esophagectomy led to the use of pathologic stage after esophagectomy as the sole basis for all cancer staging. Data analysis in the 8th edition demonstrated that simple sharing of stage groups among classifications was not possible, due to marked survival differences and unique pathologic stage groups after resection (esophagectomy or endoscopic resection) (Table 3) (5-7).

Today, pathologic staging is losing its clinical relevance for advanced-stage cancer as neoadjuvant therapy replaces esophagectomy alone. However, it remains relevant for early-stage cancers, as the most accurate reflection of cancer facts and as a survival reference point.

\section{Applications of 8th edition pathologic staging (pTNM) to clinical practice}

\section{Resection}

Before resection, the surgeon or endoscopist must confirm the epicenter of the cancer $(\mathrm{pL})$. Complete resection of the primary cancer cannot be overstated. Adequate resection requires obtaining satisfactory margins and preserving all margins, particularly the radial margin. The definition of a positive radial margin depends on the pathologic reporting system used. The Royal College of Pathologists defines an R1 (microscopically positive) margin as cancer within $1 \mathrm{~mm}$ of the margin (43); the College of American Pathologists defines R1 as microscopic involvement of the margin (44). A recent data-mining study reported that $10 \%$ of esophagectomy patients had positive resection margins (45). Increasing $\mathrm{c} T$ was associated with a positive margin. Survival is markedly diminished in incompletely 


\begin{tabular}{|c|c|c|c|c|c|}
\hline pStage group & pT & $\mathrm{pN}$ & $\mathrm{pM}$ & pGrade & pLocation \\
\hline \multicolumn{6}{|c|}{ Squamous cell carcinoma } \\
\hline 0 & Tis & NO & MO & $\mathrm{N} / \mathrm{A}$ & Any \\
\hline IA & $\mathrm{T} 1 \mathrm{a}$ & NO & MO & $\mathrm{G} 1, \mathrm{X}$ & Any \\
\hline \multirow[t]{3}{*}{ IB } & $\mathrm{T} 1 \mathrm{~b}$ & NO & MO & $\mathrm{G} 1, \mathrm{X}$ & Any \\
\hline & $\mathrm{T} 1$ & No & MO & G2-3 & Any \\
\hline & T2 & NO & MO & G1 & Any \\
\hline \multirow[t]{3}{*}{$\| A$} & T2 & NO & MO & $\mathrm{G} 2-3, \mathrm{X}$ & Any \\
\hline & T3 & NO & MO & Any & Lower \\
\hline & T3 & NO & MO & G1 & Upper/middle \\
\hline \multirow[t]{4}{*}{ IIB } & T3 & NO & MO & G2-3 & Upper/middle \\
\hline & T3 & NO & MO & $x$ & Any \\
\hline & T3 & NO & MO & Any & $x$ \\
\hline & $\mathrm{T} 1$ & N1 & MO & Any & Any \\
\hline \multirow[t]{2}{*}{ IIIA } & $\mathrm{T} 1$ & N2 & MO & Any & Any \\
\hline & T2 & N1 & MO & Any & Any \\
\hline \multirow[t]{3}{*}{ IIIB } & $\mathrm{T} 4 \mathrm{a}$ & No-1 & MO & Any & Any \\
\hline & T3 & N1 & MO & Any & Any \\
\hline & T2-3 & N2 & MO & Any & Any \\
\hline \multirow[t]{3}{*}{ IVA } & $\mathrm{T} 4 \mathrm{a}$ & N2 & MO & Any & Any \\
\hline & $\mathrm{T} 4 \mathrm{~b}$ & No-2 & MO & Any & Any \\
\hline & $\mathrm{T} 1-4$ & N3 & MO & Any & Any \\
\hline IVB & $\mathrm{T} 1-4$ & No-3 & M1 & Any & Any \\
\hline \multicolumn{6}{|c|}{ Adenocarcinoma } \\
\hline 0 & Tis & NO & MO & $\mathrm{N} / \mathrm{A}$ & \\
\hline IA & $\mathrm{T} 1 \mathrm{a}$ & NO & MO & $\mathrm{G} 1, \mathrm{X}$ & \\
\hline \multirow[t]{2}{*}{ IB } & T1a & NO & MO & G2 & \\
\hline & $\mathrm{T} 1 \mathrm{~b}$ & NO & MO & $\mathrm{G} 1-2, \mathrm{X}$ & \\
\hline \multirow[t]{2}{*}{ IC } & $\mathrm{T} 1$ & NO & MO & G3 & \\
\hline & T2 & NO & MO & G1-2 & \\
\hline IIA & T2 & NO & MO & G3, $X$ & \\
\hline \multirow[t]{2}{*}{ IIB } & $\mathrm{T} 1$ & N1 & MO & Any & \\
\hline & T3 & NO & MO & Any & \\
\hline \multirow[t]{2}{*}{ IIIA } & $\mathrm{T} 1$ & N2 & MO & Any & \\
\hline & T2 & N1 & MO & Any & \\
\hline \multirow[t]{3}{*}{ IIIB } & $\mathrm{T} 4 \mathrm{a}$ & No-1 & MO & Any & \\
\hline & T3 & N1 & MO & Any & \\
\hline & T2-3 & N2 & MO & Any & \\
\hline \multirow[t]{4}{*}{ IVA } & $\mathrm{T} 4 \mathrm{a}$ & N2 & MO & Any & \\
\hline & T4b & No-2 & MO & Any & \\
\hline & $\mathrm{T} 1-4$ & N3 & MO & Any & \\
\hline & $\mathrm{T} 1-4$ & No-3 & M1 & Any & \\
\hline
\end{tabular}

resected patients, and although adjuvant radiation is reported to improve survival, it has minimal clinical relevance.

To avoid false-positive resection margins, separation of periesophageal soft tissue in the area of the primary cancer should be avoided. Any clinical or intraoperative finding suggestive of a positive margin should prompt an intraoperative pathology consultation, with frozen section study of the resection margin in question (46). If the margin is positive, resection should be extended to obtain a negative margin if possible.

Lymphadenectomy should be based on the new AJCC 8th edition regional lymph node map (1). Lymphadenectomy sufficient to determine $\mathrm{pN}$ is different from that necessary for optimal survival; at surgery, a balance of these goals is necessary (14). More lymph nodes are required to identify the uncommon early-stage cancer with regional lymph node metastases. Increasing $\mathrm{pN}$ is associated with increasing $\mathrm{p} T$, increasing cancer length, increasing $\mathrm{G}$, and more lymph nodes resected. Optimal lymphadenectomy to classify $\mathrm{pN}$ is 60 for smaller cancers $(<2.5 \mathrm{~cm})$ and 20 for larger ones. However, a different lymphadenectomy strategy is required to provide a possible therapeutic (survival) benefit for advanced cancers (47). Optimum lymphadenectomy for maximal survival follows the simple rule of resecting 10 regional lymph nodes for $\mathrm{pT} 1$ cancers, 20 for $\mathrm{pT} 2$, and $\leq 30$ for $\mathrm{pT} 3$.

An accurate count of resected lymph nodes is important to assess quality of resection and for prognostication. If lymph nodes are fragmented at resection, the surgeon must provide the number of regional lymph nodes in the fragmented specimen.

\section{Handling of resection specimen}

Accurate pathologic staging requires careful examination of the gross specimen for cancer size, shape, configuration, location, distance from margins (proximal, distal, and radial), and nodal dissection. Inking the adventitial aspect of the specimen facilitates microscopic assessment of $\mathrm{pT}$ and $\mathrm{R}$.

Lymph node dissection is a major component of pathologic staging. Optimal lymph node staging depends on the amount of nodal tissue resected and the dissecting skills of the pathology staff. The periesophageal soft tissue (adventitia) should be thoroughly dissected. Lymph node retrieval should be performed only after full-thickness sections of the cancer and deepest extent of invasion into the adventitia have been obtained. Lack of adherence to this practice leads to false-positive radial margins. In cases 
where lymph node tissue is submitted as separate specimens, the number of lymph nodes, including presence of matted lymph nodes, should be documented in the pathology report. In specimens received in multiple fragments, accurate lymph node count is not possible if the surgeon has not documented the count, and this fact should be recorded.

The American College of Gastroenterology has endorsed EMR as a modality both for diagnosis and treatment of mucosal nodularity in patients with Barrett esophagus (48). EMR specimens provide larger, intact specimens containing submucosal tissue for accurate pathologic assessment of $\mathrm{pG}, \mathrm{pT}$, and lymphovascular invasion in patients with superficial esophageal adenocarcinoma. To facilitate accurate staging, specimens should be oriented and fixed by pinning to a corkboard and serially sectioned after inking the lateral and deep margins of the specimen. Assessing EMR specimens is challenging because specimen edges often exhibit thermal artifacts and tend to curl. This precludes accurate assessment of lateral mucosal margins. Duplication of muscularis mucosae, often seen in Barrettrelated adenocarcinomas, can result in misinterpreting invasion into the space between duplicated muscularis mucosae as submucosal invasion (49). Cancers should be categorized as pT1b only when neoplastic glands infiltrate beyond the duplicated muscularis mucosal layer, involve the plane containing submucosal glands, or are located adjacent to large-caliber arterial branches not normally found in the mucosa.

ESD is emerging as an endoscopic technique for en bloc resection of cancers. Tumors that are likely to demonstrate submucosal invasion are larger than $15 \mathrm{~mm}$ or poorly "lifting" (22). Similar to EMR specimens, the tissue orientation in ESD specimens facilitates the crucial distinction between pT1a and pT1b cancer (23).

\section{Pathologic staging: decision-making}

Theoretically, pathologic staging could facilitate decisionmaking and has the potential to provide precision cancer care in the post-esophagectomy period. However, use of this information to direct postoperative adjuvant therapy awaits more effective treatment.

\section{Pathologic staging: prognostication}

Survival according to pTNM stage group was the best distributed of all classifications, with monotonically decreasing survival with increasing subgroup and group, except for stage group 0 , which by AJCC definition was

\begin{tabular}{llll}
\multicolumn{4}{l}{ Table 4 Postneoadjuvant therapy (ypTNM) stage groups } \\
\hline ypStage group & ypT & ypN & ypM \\
\hline I & T0-2 & N0 & M0 \\
II & T3 & N0 & M0 \\
IIIA & T0-2 & N1 & M0 \\
IIIB & T4a & No & M0 \\
& T3 & N1-2 & M0 \\
& T0-3 & N2 & M0 \\
IVA & T4a & N1-2, X & M0 \\
& T4b & N0-2 & M0 \\
& T1-4 & N3 & M0 \\
IVB & T1-4 & N0-3 & M1 \\
\hline X, not defined. & & &
\end{tabular}

limited to pTis. Subgrouping maximized distinctiveness of survival between groups and subgroups. Homogeneity of survival within groups was excellent in all but the advanced groups, which would be remedied by additional higher pN subcategories, but is clinically irrelevant because survival is poor in patients with more than seven regional lymph node metastases (N3). Thus, prognostication using pathologic stage groups is the most refined of all classifications. Personalized prognostication is afforded with additional cancer and patient variables in the prediction model.

\section{8th edition postneoadjuvant staging}

New to the 8th edition is stage grouping of patients who have undergone neoadjuvant therapy and pathologic review of the resection specimen. Drivers of this addition include absence of equivalent pathologic (pTNM) categories for the peculiar postneoadjuvant categories ypT0N0-3M0 and ypTisN0-3M0, dissimilar stage group compositions, and markedly different survival profiles. Survival for ypTNM groups differs from that for comparable pTNM groups: ypTNM survival is less distinctive between groups, and survival curves are greatly depressed from above, with much poorer survival of early ypTNM groups compared with corresponding pTNM groups and dismal survival of advanced ypTNM groups, no better or worse than corresponding pTNM groups. The groupings are identical for both cell types (Table 4). 


\section{Applications of 8th edition postneoadjuvant staging (ypTNM) to clinical practice}

\section{Resection}

Just as for pTNM, adequate resection with preservation of margins and adequate lymphadenectomy are essential. That lymphadenectomy has not been demonstrated to affect survival in patients undergoing resection after preoperative therapy $(4,50)$ should not influence extent of lymphadenectomy.

\section{Handling of resection specimen}

Gross appearance of a cancer may vary depending on response to neoadjuvant therapy. With minimal response, the cancer is readily visualized and is sampled similarly to a non-treated cancer or cancer treated by esophagectomy alone. With a good response, the cancer may show only ulceration or mucosal irregularity. The cancer bed should be completely submitted for histologic evaluation.

Obliteration of anatomic landmarks poses diagnostic challenges in assigning ypT, especially for EGJ cancers (51). In some institutions, for EGJ cancers, the esophageal adventitial surface and gastric serosa are inked with different colors to determine the exact anatomic location and ypT (52). This practice will be obviated with genetic signature determination of cancer cell of origin.

Neoadjuvant therapy induces several histologic changes, including ulceration, mural fibrosis, acellular mucin pools, and dystrophic calcification. Cancer cells must be distinguished from reactive stromal cells and macrophages. Regardless of the cell type, residual cancer cells usually demonstrate enlarged, irregular, and hyperchromatic nuclei with a dense homogeneous nuclear chromatin pattern and abundant cytoplasm. Occasionally, residual cancer cells show neuroendocrine phenotype or squamous features. These foci should be considered when determining ypT (53).

Neoadjuvant histopathologic changes may preclude accurate grading of cancer, especially in cases with minimal residual cancer. This underscores the importance of grading cancers on preoperative biopsy. Acellular mucin pools should not be used to determine p T or R (53).

Cancer regression grading as described by Mandard et al. is the most widely used system to assess response to therapy (54). The three-tiered cancer regression grading system outlined by Ryan et al. for assessing treated rectal cancer has shown good interobserver reproducibility among pathologists and is incorporated in the College of American Pathologists templates (55).
In patients receiving neoadjuvant therapy, lymph nodes can atrophy and be difficult to recognize macroscopically. In these cases, histologic assessment of most of the periesophageal soft tissue is helpful to retrieve grossly impalpable lymph nodes. After treatment, lymph node parenchyma shows fibrosis, lymphoid depletion, and acellular mucin lakes. Lymph nodes with these changes, and without any viable cancer cells, should be considered negative for metastasis (ypN0). Immunohistochemical stains such as cytokeratin AE1/AE3 may be used to confirm the presence of rare residual cancer cells. However, because false-positive results may occur, they should be interpreted in conjunction with morphologic findings.

\section{Postneoadjuvant staging: decision-making}

The role of yp TNM in additional treatment planning is currently limited. However, for adenocarcinoma, addition of adjuvant chemotherapy provides a survival benefit in patients with residual nodal disease $(y p N+)$ (56). To realize precision cancer care, advances are necessary in both targeted neoadjuvant and adjuvant therapies.

\section{Postneoadjuvant staging: prognostication}

With the introduction of 8 th edition ypTNM cancer staging, prognostication is specific for patients undergoing neoadjuvant therapy and is not shared with any other classification.

\section{Acknowledgements}

None.

\section{Footnote}

Conflicts of Interest: The authors have no conflicts of interest to declare.

\section{References}

1. Rice TW, Kelsen DP, Blackstone EH, et al. Esophagus and esophagogastric junction. In: Amin MB, Edge SB, Greene FL, et al., editors. AJCC Cancer Staging Manual, 8th ed. New York: Springer, 2017:185-202.

2. Rice TW, Chen LQ, Hofstetter WL, et al. Worldwide Esophageal Cancer Collaboration: pathologic staging data. Dis Esophagus 2016;29:724-33.

3. Rice TW, Apperson-Hansen C, DiPaola LM, et al. Worldwide Esophageal Cancer Collaboration: clinical 
staging data. Dis Esophagus 2016;29:707-14.

4. Rice TW, Lerut TE, Orringer MB, et al. Worldwide Esophageal Cancer Collaboration: neoadjuvant pathologic staging data. Dis Esophagus 2016;29:715-23.

5. Rice TW, Ishwaran H, Hofstetter WL, et al. Recommendations for pathologic staging (pTNM) of cancer of the esophagus and esophagogastric junction for the 8th edition AJCC/UICC staging manuals. Dis Esophagus 2016;29:897-905.

6. Rice TW, Ishwaran H, Blackstone EH, et al. Recommendations for clinical staging (cTNM) of cancer of the esophagus and esophagogastric junction for the 8th edition AJCC/UICC staging manuals. Dis Esophagus 2016;29:913-9.

7. Rice TW, Ishwaran H, Kelsen DP, et al. Recommendations for neoadjuvant pathologic staging (ypTNM) of cancer of the esophagus and esophagogastric junction for the 8th edition AJCC/UICC staging manuals. Dis Esophagus 2016;29:906-12.

8. Cancer Genome Atlas Research Network. Comprehensive molecular characterization of gastric adenocarcinoma. Nature 2014;513:202-9.

9. Hayakawa Y, Sethi N, Sepulveda AR, et al. Oesophageal adenocarcinoma and gastric cancer: should we mind the gap? Nat Rev Cancer 2016;16:305-18.

10. Montgomery E, Field JK, Boffetta P. Squamous cell carcinoma of the oesophagus. In: Bosman FT, Carneiro F, Hruban RH, et al., editors. WHO Classification of Tumours of the Digestive System, 4th edition. Lyon: International Agency for Research on Cancer, 2010:18-24.

11. Flejou JF, Odze RD, Montgomery E, et al. Adenocarcinoma of the oesophagus. In: Bosman FT, Carneiro F, Hruban RH, et al., editors. WHO Classification of Tumours of the Digestive System, 4th edition. Lyon: International Agency for Research on Cancer, 2010:25-31.

12. Chirieac LR, Swisher SG, Correa AM, et al. Signetring cell or mucinous histology after preoperative chemoradiation and survival in patients with esophageal or esophagogastric junction adenocarcinoma. Clin Cancer Res 2005;11:2229-36.

13. Kelly S, Harris KM, Berry E, et al. A systematic review of the staging performance of endoscopic ultrasound in gastro-oesophageal carcinoma. Gut 2001;49:534-9.

14. Rice TW, Ishwaran H, Hofstetter WL, et al. Esophageal Cancer: Associations With (pN+) Lymph Node Metastases. Ann Surg 2017;265:122-9.

15. Barbour AP, Rizk NP, Gerdes H, et al. Endoscopic ultrasound predicts outcomes for patients with adenocarcinoma of the gastroesophageal junction. J Am Coll Surg 2007;205:593-601.

16. Blackshaw G, Lewis WG, Hopper AN, et al. Prospective comparison of endosonography, computed tomography, and histopathological stage of junctional oesophagogastric cancer. Clin Radiol 2008;63:1092-8.

17. Murata Y, Napoleon B, Odegaard S. High-frequency endoscopic ultrasonography in the evaluation of superficial esophageal cancer. Endoscopy 2003;35:42935; discussion 436.

18. Puli SR, Reddy JB, Bechtold ML, et al. Staging accuracy of esophageal cancer by endoscopic ultrasound: a metaanalysis and systematic review. World J Gastroenterol 2008;14:1479-90.

19. Rice TW, Mason DP, Murthy SC, et al. T2N0M0 esophageal cancer. J Thorac Cardiovasc Surg 2007;133:317-24.

20. Hardacker TJ, Ceppa D, Okereke I, et al. Treatment of clinical T2N0M0 esophageal cancer. Ann Surg Oncol 2014;21:3739-43.

21. Thota PN, Sada A, Sanaka MR, et al. Correlation between endoscopic forceps biopsies and endoscopic mucosal resection with endoscopic ultrasound in patients with Barrett's esophagus with high-grade dysplasia and early cancer. Surg Endosc 2017;31:1336-41.

22. Pimentel-Nunes P, Dinis-Ribeiro M, Ponchon T, et al. Endoscopic submucosal dissection: European Society of Gastrointestinal Endoscopy (ESGE) Guideline. Endoscopy 2015;47:829-54.

23. Yang D, Coman RM, Kahaleh M, et al. Endoscopic submucosal dissection for Barrett's early neoplasia: a multicenter study in the United States. Gastrointest Endosc 2016. [Epub ahead of print].

24. Gress DM, Edge SB, Greene FL, et al. Principles of Cancer Staging. In: Amin MB, Edge SB, Greene FL, et al., editors. AJCC Cancer Staging Manual, 8th ed. New York: Springer, 2017:3-30.

25. Kutup A, Link BC, Schurr PG, et al. Quality control of endoscopic ultrasound in preoperative staging of esophageal cancer. Endoscopy 2007;39:715-9.

26. van Overhagen H, Becker CD. Diagnosis and staging of carcinoma of the esophagus and gastroesophageal junction, and detection of postoperative recurrence, by computed tomography. In: Meyers M, editor. Neoplasms of the digestive tract. Imaging, staging and management. Philadelphia: Lippincott-Raven, 1998:31-48.

27. Doi N, Aoyama N, Tokunaga M, et al. Possibility of pre- 
operative diagnosis of lymph node metastasis based on morphology. Hepatogastroenterology 1999;46:977-80.

28. van Vliet EP, Heijenbrok-Kal MH, Hunink MG, et al. Staging investigations for oesophageal cancer: a metaanalysis. Br J Cancer 2008;98:547-57.

29. Flanagan FL, Dehdashti F, Siegel BA, et al. Staging of esophageal cancer with $18 \mathrm{~F}$-fluorodeoxyglucose positron emission tomography. AJR Am J Roentgenol 1997;168:417-24.

30. Kato H, Kuwano H, Nakajima M, et al. Comparison between positron emission tomography and computed tomography in the use of the assessment of esophageal carcinoma. Cancer 2002;94:921-8.

31. Flamen P, Lerut A, Van Cutsem E, et al. Utility of positron emission tomography for the staging of patients with potentially operable esophageal carcinoma. J Clin Oncol 2000;18:3202-10.

32. Roedl JB, Blake MA, Holalkere NS, et al. Lymph node staging in esophageal adenocarcinoma with PET-CT based on a visual analysis and based on metabolic parameters. Abdom Imaging 2009;34:610-7.

33. Choi J, Kim SG, Kim JS, et al. Comparison of endoscopic ultrasonography (EUS), positron emission tomography (PET), and computed tomography (CT) in the preoperative locoregional staging of resectable esophageal cancer. Surg Endosc 2010;24:1380-6.

34. Wiersema MJ, Vilmann P, Giovannini M, et al. Endosonography-guided fine-needle aspiration biopsy: diagnostic accuracy and complication assessment. Gastroenterology 1997;112:1087-95.

35. Bergman JJ. The endoscopic diagnosis and staging of oesophageal adenocarcinoma. Best Pract Res Clin Gastroenterol 2006;20:843-66.

36. Giovannini M, Seitz JF, Monges G, et al. Fine-needle aspiration cytology guided by endoscopic ultrasonography: results in 141 patients. Endoscopy 1995;27:171-7.

37. Natsugoe S, Yoshinaka H, Shimada M, et al. Number of lymph node metastases determined by presurgical ultrasound and endoscopic ultrasound is related to prognosis in patients with esophageal carcinoma. Ann Surg 2001;234:613-8.

38. Chen J, Xu R, Hunt GC, et al. Influence of the number of malignant regional lymph nodes detected by endoscopic ultrasonography on survival stratification in esophageal adenocarcinoma. Clin Gastroenterol Hepatol 2006;4:573-9.

39. Twine CP, Roberts SA, Rawlinson CE, et al. Prognostic significance of the endoscopic ultrasound defined lymph node metastasis count in esophageal cancer. Dis Esophagus 2010;23:652-9.

40. Li Z, Rice TW. Diagnosis and staging of cancer of the esophagus and esophagogastric junction. Surg Clin North Am 2012;92:1105-26.

41. Luketich JD, Friedman DM, Weigel TL, et al. Evaluation of distant metastases in esophageal cancer: 100 consecutive positron emission tomography scans. Ann Thorac Surg 1999;68:1133-6; discussion 1136-7.

42. International Union Against Cancer (UICC). TNM Classification of Malignant Tumours. Geneva; 1968.

43. The Royal College of Pathologists. Dataset for the histopathological reporting of oesophageal carcinoma, 2nd edition. London: The Royal College of Pathologists, 2007.

44. College of American Pathologists. Protocol for the examination of specimens from patients with carcinoma of the oesophagus. Northfield: College of American Pathologists, 2009. Available online: goo.gl/lytw6I

45. Javidfar J, Speicher PJ, Hartwig MG, et al. Impact of Positive Margins on Survival in Patients Undergoing Esophagogastrectomy for Esophageal Cancer. Ann Thorac Surg 2016;101:1060-7.

46. Liu X, Rice TW, Xiao SY, et al. Diagnostic problems during esophageal and gastric surgery. In: Marchevsky AM, Balzer BL, Abdul-Karim FW, editors. Intraoperative consultation. Philadelphia: Elsevier, 2015:154-68.

47. Rizk NP, Ishwaran H, Rice TW, et al. Optimum lymphadenectomy for esophageal cancer. Ann Surg 2010;251:46-50.

48. Shaheen NJ, Falk GW, Iyer PG, et al. ACG Clinical Guideline: Diagnosis and Management of Barrett's Esophagus. Am J Gastroenterol 2016;111:30-50; quiz 51.

49. Lewis JT, Wang KK, Abraham SC. Muscularis mucosae duplication and the musculo-fibrous anomaly in endoscopic mucosal resections for barrett esophagus: implications for staging of adenocarcinoma. Am J Surg Pathol 2008;32:566-71.

50. Koen Talsma A, Shapiro J, Looman CW, et al. Lymph node retrieval during esophagectomy with and without neoadjuvant chemoradiotherapy: prognostic and therapeutic impact on survival. Ann Surg 2014;260:78692; discussion 792-3.

51. Chang F, Deere H, Mahadeva U, et al. Histopathologic examination and reporting of esophageal carcinomas following preoperative neoadjuvant therapy: practical guidelines and current issues. Am J Clin Pathol 2008;129:252-62.

52. Wu TT, Chirieac LR, Abraham SC, et al. Excellent 
interobserver agreement on grading the extent of residual carcinoma after preoperative chemoradiation in esophageal and esophagogastric junction carcinoma: a reliable predictor for patient outcome. Am J Surg Pathol 2007;31:58-64.

53. Hornick JL, Farraye FA, Odze RD. Prevalence and significance of prominent mucin pools in the esophagus post neoadjuvant chemoradiotherapy for Barrett's-associated adenocarcinoma. Am J Surg Pathol 2006;30:28-35.

54. Mandard AM, Dalibard F, Mandard JC, et al. Pathologic assessment of tumor regression after preoperative

Cite this article as: Rice TW, Patil DT, Blackstone EH. 8th edition AJCC/UICC staging of cancers of the esophagus and esophagogastric junction: application to clinical practice. Ann Cardiothorac Surg 2017;6(2):119-130. doi: 10.21037/ acs.2017.03.14 chemoradiotherapy of esophageal carcinoma.

Clinicopathologic correlations. Cancer 1994;73:2680-6.

55. Ryan R, Gibbons D, Hyland JM, et al. Pathological response following long-course neoadjuvant chemoradiotherapy for locally advanced rectal cancer. Histopathology 2005;47:141-6.

56. Burt BM, Groth SS, Sada YH, et al. Utility of Adjuvant Chemotherapy After Neoadjuvant Chemoradiation and Esophagectomy for Esophageal Cancer. Ann Surg 2016. [Epub ahead of print]. 